\title{
El cambio en la política exterior de Estados Unidos que permitió el desarrollo del uso pacífico de energía nuclear en Argentina*
}

por Adrián Contursi Reynoso ${ }^{* *}$

\section{- Resumen}

Las bombas atómicas arrojadas sobre Japón no significan sólo el fin de la Segunda Guerra Mundial en 1945. También es el punto de partida para el creciente interés de los países periféricos para obtener dicha tecnología, aunque no desde un enfoque bélico sino como oportunidad de desarrollo. El presente trabajo explora el cambio de la política de cooperación estadounidense en referencia a los conocimientos sobre la energía nuclear y cómo fue la relación de Argentina con Estados Unidos durante su propio proceso de desarrollo nuclear, hasta la concreción del primer reactor RA-1.

\section{- Palabras Clave}

Energía Nuclear, Argentina, Uso pacífico.

\section{Inglés | English}

The change on the foreign policy of the United States of America wich allowed the development of pacific use of nuclear energy in Argentina.

\section{- Abstract}

The atomic bombs launched over Japan do not mean only the end of the Second World War in 1945. This is also the starting point for growing interest of the peripheries countries to obtain such technology. The focus of these countries is not warlike but a development opportunity. The current paper explores the change in the foreign cooperation policy of the United States linked to the knowledge of nuclear energy and the relation between Argentina and the United States during the process of nuclear development of the southern country until the building of the first nuclear reactor RA-1.

\section{- Key words}

Nuclear Energy, Argentina, Pacific use.

\footnotetext{
* Ponencia presentada en el XII Congreso Nacional y V Internacional sobre Democracia. En la Mesa Redonda: Cooperación Internacional en la Agenda Estratégica Regional y Argentina, el 15 de Septiembre de 2016.

** Licenciado en Ciencia Política por la Universidad Nacional del Litoral. Maestrando en Integración y Cooperación por el Centro de Estudios Interdisciplinarios de la Universidad Nacional de Rosario. acontursi@gmail.com
} 


\section{La bomba atómica y el día después de la destrucción}

Harry Truman, $33^{\circ}$ Presidente de los EE. UU., es recordado principalmente por ser quién ordenó el ataque a Japón con bombas atómicas como consecuencia directa de que aquel país no acatara el ultimátum de rendición enviado por los aliados desde la Conferencia de Potsdam, Alemania, el 2 de Agosto de 1945.

El 6 de Agosto de ese año, la bomba atómica de uranio, conocida como Little Boy, impactó en Hiroshima. Tres días después, el 9 de Agosto, una segunda bomba atómica, Fat Man, fue lanzada sobre Nagasaki. El Emperador Hirohito aceptó los términos de rendición de Potsdam el 15 de Agosto de 1945, finalizando así la Segunda Guerra Mundial.

Al día siguiente de la explosión en Nagasaki, Yosuke Yamahata, fotógrafo del ejército japonés, fue asignado junto a otros dos soldados para documentar los efectos de la Bomba Atómica. Dentro de la extensiva galería fotográfica, una de ellas es fácilmente reconocible, un Torii erecto en el medio de la devastación. Un Torii es un pórtico tradicional que marca la frontera entre lo profano y lo sagrado y suelen anticipar el ingreso a santuarios sintoísta. El Torii del Santuario Sanno, a solo 800 metros del hipocentro de la explosión se mantuvo en pie. Esta imagen sería posteriormente asociada como símbolo de resistencia y reconstrucción.

Mientras tanto, en los EE. UU. se presentaba a la bomba atómica como la entrada en una nueva era tecnológica.

“...uno de los hitos científicos del siglo ha sido atravesado, y la "era de la energía atómica", la cual puede ser una fuerza tremenda para el avance de la civilización también como para la destrucción, estaba al alcance de la mano."' (SHALETT, 1945:1)

La tensión dialéctica, según Hurtado (2014), combinaba el miedo a la destrucción con la esperanza de una nueva fuente de energía. Significaba también el comienzo de la Guerra Fría.

\section{Subdesarrollo, semiperiferia y autono- mía}

Immanuel Wallerstein (2005) expresa que el sistema histórico tiene vidas que en algún punto en el tiempo y el espacio manifiestan su existencia. Si sobreviven a su nacimiento, vivirán su existencia histórica dentro de estructuras que las contienen y moldean y también son moldeadas. Este ciclo se repite constantemente sin percatarse de las contradicciones propias del sistema. Todo esto implica que el sistema se encuentre con problemas que no puede resolver, y que se constituirán crisis sistémicas. Pero, aunque no puedan ser resueltas, soluciones parciales o bifurcaciones del sistema permiten la continuidad o reconstrucción del mismo.

“...el sistema se enfrenta con dos soluciones alternativas para su crisis, ambas son intrínsecamente posibles. En efecto, los miembros del sistema son convocados para hacer una elección histórica sobre cuál de los caminos alternativos seguirán, eso es, qué tipo de nuevo sistema será construido." (WALLERSTEIN, 2005:2)

Pero, no es claro cuál camino realmente escogerán los miembros del sistema. El proceso de elección es caótico y el sistema se presenta en una amplia oscilación. Aun así, eventualmente tiende hacia una dirección en específico.

Después de la Segunda Guerra Mundial, existió un crecimiento económico general para el sistema mundial. No obstante, Wallerstein (2005) especifica que la brecha entre el centro y la periferia se agrandó. Nuevos estratos privilegiados emergieron. Podríamos identificar que el conocimiento sobre la energía nuclear constituyó uno de los reductos privilegiados, en donde el centro y la periferia estaban completamente distanciados.

Por otro lado, Cardoso y Faletto (1972) plantearon esta dicotomía en conceptos antepuestos como desarrollo-subdesarrollo, centro-periferia y autonomía-dependencia. Domingues (2012) explica que estos pares de conceptos corresponden a diferentes posiciones y relaciones de los países. El primero refiere a la economía interna de cada país y su comparación dentro del sistema internacional, el segundo par es relativo a la función de cada país en el

1.- Traducción del inglés hecha por el autor. 
proceso económico internacional: producción industrial, producción agrícola, minería. El tercer par indica la capacidad de autodeterminación de cada país en relación a fuerzas externas.

Wallerstein (1984) introduce el concepto de semiperiferia, en donde algunos Estados se acercan a la estructura productiva de los países centrales, logrando que los mismos reproduzcan las conductas de relación con los países de la periferia. Pero, la semiperiferia no constituye una posición estática sino un lugar fluctuante entre el ascenso y descenso del país en la división internacional del trabajo.

\section{Estados Unidos y el monopolio del co- nocimiento}

$\mathrm{Al}$ año siguiente de las explosiones nucleares, EE. UU. prohibió la difusión de información referida a su programa nuclear, a través de la Ley 585 del $1^{\circ}$ de Agosto de 1946, conocida como Ley McMahon, por el Senador Brien McMahon quien fue uno de los principales impulsores.

"La importancia de la bomba atómica para propósitos militares es evidente. Los efectos del uso de la energía atómica para propósitos civiles en cuanto a estructuras sociales, económicas y políticas no puede ser hoy determinada. (...) Es razonable anticipar, que de cualquier modo, debemos intervenir ahora sobre una fuente de energía que causará profundos cambios en nuestro presente modo de vida." ${ }^{2}$ (Ley 585; 1946)

El Congreso estadounidense logró comprobar que información relacionada a su proyecto nuclear, denominado Manhattan, había sido filtrada a espías soviéticos. Como consecuencia, la Ley McMahon fue aprobada, clasificando toda la información referida al hecho atómico. En el mismo acto, se creaba USA EC (United Status Atomic Energy Commission), el ente civil a cargo de la Energía Atómica, aunque con una estrecha relación con el sector militar.

$\mathrm{Al}$ impedir compartir información sobre su programa nuclear, EE. UU. trató de bloquear la diseminación del conocimiento sobre el átomo, incluso hasta con sus aliados más cercanos como el Reino Unido y Canadá. Hasta ese momento, EE. UU. era el único país que había logrado producir una bomba atómica, lo que lo colocaba en una posición única que pretendía mantener durante el mayor tiempo posible, aproximadamente veinte años según sus propias proyecciones.

Sin embargo, inmediatamente se vislumbró que eso sería imposible. La restricción de la Ley McMahon impulsó a que los países emprendieran sus propios programas de desarrollo nuclear y la Unión Soviética logró construir una bomba nuclear en 1949, demostrándolo cabalmente al realizar una explosión de prueba. También Gran Bretaña alcanzó el objetivo de la bomba en octubre de 1952. EE.UU. ya no era el único país con capacidad nuclear.

\section{Una oportunidad para el desarrollo de Argentina}

En Octubre de 1947, Juan Domingo Perón comunicaba el lanzamiento del primer Plan Quinquenal, con el objetivo de que el país adquiriese autonomía económica. Para ello, se implementó un proceso de estatización de empresas extranjeras: telecomunicaciones, ferrocarriles, elevadores, servicios de gas, entre otras. Posteriormente, la Reforma Constitucional de 1949, en su artículo 40, especificaba que el Estado tenía ante la actividad económica la facultad de intervenirla y monopolizar ciertas tareas, así como fijar los precios de las expropiaciones, declaraba como irrenunciables del Estado la función del comercio exterior y los servicios públicos. Y, especificaba que los Minerales y fuentes de energía, son propiedad inalienable de la $\mathrm{Na}$ ción. Esto fue entendido como la nacionalización de los yacimientos de hidrocarburos y, también, de las reservas de uranio. Argentina apostaba al autoabastecimiento energético:

"...luego de las explosiones de Hiroshima y Nagasaki, un sector de las Fuerzas Armadas vio en el desarrollo del área atómica una posible solución a la dependencia de carbón y petróleo extranjero, factor percibido como rasgo central de la vulnerabilidad económica del país." (HURTADO, 2014:39) 
El camino elegido incluía el desarrollo del área científica para hacer frente al déficit y la energía nuclear había sido prontamente identificada como un camino posible.

Pero, las relaciones diplomáticas entre Argentina y Estados Unidos durante el primer gobierno de Perón se encontraban en tensión. Principalmente, porque las políticas de Perón tendían a evitar involucrarse con organismos internacionales como el Banco Mundial y el Fondo Monetario Internacional, los cuales eran ampliamente reconocidos como foros internacionales de los EE. UU. La postura de evitar tener que ceder autonomía en la toma de decisiones a cambio de préstamos llevó a Argentina a un cierto grado de distanciamiento con los Estados Unidos y de aislamiento internacional. Cabe recordar que EE. UU. era el país que encabezaba la tecnología nuclear en aquella época y la relación bilateral deteriorada impedía una cooperación técnica acorde.

Hurtado (2014), permite ver con claridad cómo diferentes medios de prensa calificaban a las aspiraciones argentinas sobre la temática nuclear como una potencial amenaza. Comenzando el 24 de Febrero de 1947 con un artículo escrito por William Mizelle en The New Republic titulado Exclusive - Peron's Atomic Plans, el cual aseveraba que Argentina estaba desarrollando un plan militar para obtener armas nucleares.

Enrique Gaviola, quien fuera el primer presidente de la Asociación de Físicos Argentinos (AFA), veía en el desarrollo nuclear una oportunidad para promover el desarrollo de la física experimental. Gaviola respondió públicamente al artículo de Mizelle, negando sus argumentos.

A pesar de la respuesta, The New Republic publicó un nuevo artículo de Mizelle el 31 de Marzo de 1947, titulado More about Peron's Atom Plans, el cual desprestigiaba la respuesta del físico y reafirmaba que Argentina se encontraba en el camino de obtener un arma atómica.

A pesar del contexto desfavorable, el físico argentino Enrique Gaviola consideraba que era un momento oportuno para que Argentina recibiera a científicos extranjeros europeos que sufrían las penurias de la postguerra. Por su recomendación, el científico alemán Werner Heisenberg fue invitado a la Argentina. Pero éste debía obtener autorización de los aliados para viajar al país y dicho permiso no fue otorgado.
"En una carta al jefe del Estado Mayor General de la Armada, con fecha del 11 de marzo, Gaviola reconocía que el fracaso de las negociaciones se debía a la interferencia de las autoridades de ocupación británicas. Sin embargo, Gaviola increpaba a la Armada por no haber mostrado interés y no hacer honor a los compromisos asumidos." (HURTADO, 2014:46)

A pesar de este inconveniente, el alemán Kart Tank, reconocido ingeniero abocado al diseño aeronáutico, realizó una recomendación de contactar a otro científico. Como resultado, en agosto de 1948 Perón conoció al austríaco Ronald Richter, quién lo convenció de iniciar el desarrollo del área nuclear, con la posibilidad de realizar una fusión controlada para obtener energía.

En Junio de 1949 comenzó la construcción de un laboratorio en la Isla Huemul, cercana a la localidad de San Carlos de Bariloche, provincia de Río Negro. Richter había convencido a Perón para que apoyara ampliamente su proyecto, sin importar sus excéntricos pedidos.

"Un hecho que contrarió desde el principio los planes del gobierno fue la negativa de Richter de incorporar investigadores argentinos." (HURTADO, 2014:54)

Para otorgarle un marco institucional, la Comisión Nacional de Energía Atómica (CNEA) fue creada por Decreto 10.935 el 31 de Mayo de 1950. En los fundamentos del mismo se expresó que la energía nuclear podría representar para Argentina una modificación sin precedentes de su matriz energética, al igual que indicaba que los intereses sobre el estudio del átomo eran pura y exclusivamente pacíficos.

"Que la energía atómica puede reemplazar a las formas corrientes de energía y que este hecho podría alterar el equilibrio económico y social del país en razón de las profundas modificaciones que determinaría en la actividad de la industria, de los transportes, de la minería, etc., por lo cual es conveniente que el Estado tome medidas de previsión correspondientes. [...]

Que la República Argentina, despreocupada de toda intención ofensiva, puede trabajar en este orden de cosas también con elevado sentido de paz en beneficio de la humanidad". (Decreto 10.936/1950) 


\section{De la gran quimera al instituto de física}

Durante una conferencia de prensa celebrada el 24 de marzo de 1951 en la Casa Rosada, Perón comunicó que se habían alcanzado reacciones termonucleares bajo condiciones de control en escala técnica.

El anuncio constituía un gran logro para la Argentina, la cual supuestamente ingresaba por la puerta grande al selecto club de países que poseían el dominio del átomo. Perón reafirmó que el desarrollo nuclear de Argentina tenía fines pacíficos y sería un aporte al crecimiento del país. Este anuncio fue recibido con escepticismo a nivel internacional. Heisenberg desconfiaban de la veracidad de los resultados anunciados, debido a que Richter no había realizado ninguna publicación científica sobre su experimento. Incluso Otto Hahn, el químico alemán que descubrió la fisión nuclear del uranio y el torio, desconocía a Richter.

En tanto, como indica Hurtado (2014), la relación entre el Secretario General de la CNEA, el coronel Enrique González y Richter entraba en conflicto por la falta de resultados de éste último y sus constantes exigencias. Finalmente, González renunciaba en febrero de 1952 y era reemplazado por el capitán de Fragata Pedro Iraolaoitía, lo que significó que el sector atómico bajo control del Ejército pasaba al control de la Armada, influencia que se extendería por tres décadas.

Iraolaoitía, con autorización de Perón, ordenó la conformación de dos comisiones para realizar pericias científicas para corroborar los resultados enunciados por Richter. Los dictámenes de las comisiones fueron contundentes, ambas concluyeron que Richter no había logrado lo que aseveró.

"De las comprobaciones efectuadas durante el funcionamiento del reactor se sigue que no existe ningún elemento de juicio que permita afirmar que una reacción de carácter nuclear se produce realmente." (BALSEIRO, 1952)

Como desenlace, se clausuró el 22 de noviembre de 1952 el proyecto Huemul. Perón había anunciado, sin saberlo, una gran mentira al mundo.

El proyecto Huemul solo puede considerarse como un gran fracaso. Años perdidos y millones invertidos en equipos costosos, como el Sincrociclotrón y el Acelerador Cockroft-Walton adquiridos a la empresa Philips Works de Eindhoven de Holanda, por recomendación de Richter. Pero, cuyo resultado no fue más que la decepción de un enunciado que no fue más que una mentira.

A pesar de ello, a sugerencia de Balseiro, los equipos fueron removidos de la isla y llevados a Bariloche. Allí fueron incorporados a un Instituto de Física que se estaba instalando, el cual con los años recibiría el nombre del propio Balseiro. El Instituto de Física comenzó a reclutar a los mejores estudiantes y egresados de física del país, creando un polo de desarrollo tecnológico sin precedentes para la Argentina.

\section{Átomos para la paz y el giro en la coo- peración internacional de lo Estados Unidos}

El 8 de Diciembre de 1953, el nuevo presidente de los EE. UU., Dwight Eisenhower, se presentaba ante la Asamblea General de las Naciones Unidas el programa Átomos para la Paz.

En su alocución, Eisenhower explicó abiertamente que los EE. UU. poseían un extenso armamento nuclear y que tanto Gran Bretaña y Canadá, sus aliados, conocían los secretos del desarrollo atómico. Además que la Unión Soviética también había logrado detonar un arma nuclear, en sus recientes pruebas. Pero añadió que la utilización en general del armamento nuclear podría significar la destrucción de civilizaciones, la aniquilación de nuestra herencia cultural.

“...es con el libro de la historia, y no con páginas aisladas, que los Estados Unidos siempre han deseado ser identificados. Mi país quiere ser constructivo, no destructivo. Quiere acuerdos, no guerras entre naciones. Quiere vivir en libertad y en la confianza que las personas de todas las naciones disfrutan igualmente del derecho a elegir sus propias formas de vida." (EISENHOWER, 1953:4)

El presidente puntualizó que esperaban que una Agencia Internacional de Energía Atómica se creara bajo el amparo de las Naciones Unidas, y que contribuyera a controlar todo el material fisionable y el uranio en manos de las diferentes naciones, así como establecer un sistema de inspección y control y que los Estados Unidos estarían honrados de ser los depositarios de tal responsabilidad. 
Claramente las intenciones de Estados Unidos representaban un cambio de estrategia. Al no poder controlar el conocimiento sobre la bomba nuclear, era preferible supervisar el desarrollo de los diferentes programas en el mundo, evitando que derivasen en la adquisición de nuevo armamento bélico.

Este giro en la cooperación internacional de Estados Unidos en temas nucleares fue fundamental para que países interesados en la temática, pero sin aspiraciones bélicas, pudieran acceder a información vital, así como a material fisionable.

Medhurst (citado en HURTADO, 2014), explicitaba que si Estados Unidos pudiera establecer su presencia nuclear en varios países, estos serían irremediablemente dependientes de la tecnología norteamericana.

Por lo cual, Átomos para la Paz también era una ventana comercial para las industrias estadounidenses, con la posibilidad de explotar comercialmente un rubro de alto valor agregado y casi sin competencia:

“...el uso de radioisótopos, junto con la promoción de los reactores de investigación, iban a ser la columna vertebral de la colaboración internacional en los usos pacífcos de la energía atómica, por lo menos hasta comienzos de los años sesenta, cuando comenzaron a comercializarse los primeros reactores para la producción de electricidad." (HURTADO, 2014:74-75)

Argentina fue el principal receptor de radioisótopos de América Latina a principios del programa. La USAEC firmó, en la segunda mitad de la década de 1950, veintiocho acuerdos cooperación en temas nucleares. Argentina, hizo lo propio el 29 de julio de 1955 en un acuerdo bilateral firmado en Washington D.C. en donde EE. UU. se comprometía a proveer uranio enriquecido a la Argentina para futuros reactores de investigación. Este acuerdo daría frutos en un futuro no muy lejano.

\section{La revolución libertadora}

En septiembre de 1955, se producía el Golpe Militar a la Presidencia de Juan Domingo Perón, que sería conocido como La Revolución Libertadora.

Como era de esperarse, el Gobierno de Facto modificó las prioridades del gobierno derrocado, lo que significó una reducción considerable del apoyo del Estado hacia la industria nacional. Esto también afectó al Instituto de Física en Bariloche, el cual no podía costear su propio mantenimiento. Durante esa fase, Balseiro se vio obligado a obtener apoyo de organismos internacionales. Diferentes profesores extranjeros fueron invitados a dar seminarios cortos ante la ausencia de un plantel propio y el apoyo fue principalmente desde la Organización Internacional de Energía Atómica (OIEA), la Organización de Estados Americanos (OEA) y las Universidades de Vancouver (Canadá) y Stanford (EE. UU.).

La OIEA había sido creada por el impulso del programa Átomos para la Paz de Eisenhower. En 1956 EE. UU. Y Reino Unido presentaron formalmente la iniciativa a la Asamblea General, la cual fue respalda por varios países, entre los cuales Argentina se encontraba presente. La OIEA alcanzó estatus formal en 1957. Esto muestra un cambio radical del nuevo gobierno en referencia a integrar los organismos internacionales que eran impulsados por los Estados Unidos.

\section{Fin de un período y comienzo de nue- vos desafíos: la génesis del RA-1}

Con el nuevo Gobierno, el Contralmirante Ingeniero Oscar Armando Quihillat asumió al frente de la CNEA. Inmediatamente, Quihillat anunció la compra del primer reactor nuclear de investigación a los Estados Unidos. Una clara muestra de que las relaciones bilaterales entre ambos países comenzaban a entrelazarse nuevamente.

“...considerando que entre 1956 y 1959 EE. UU. iba a exportar alrededor de cuarenta reactores de investigación, parece claro que la Argentina era un país más dentro de la esfera de influencia de la agresiva política exterior norteamericana para avanzar sobre el incipiente mercado de reactores de investigación. Como parte del "Atoms-for-Peace Program Grants for Research Reactors", EE. UU. concedía al país comprar un subsidio de 350.000 dólares." (HURTADO, 2014:84)

Quinhillat viajó a New York para efectivizar la compra pero se encontró con algunas demoras legales en la adquisición. Mientras se resolvían los trámites, viajó a una conferencia en Filadelfia donde se discutía la conveniencia de utilizar uranio natural o uranio enriquecido. Según relata 
Carlos Domingo (2003), Quinhillat se encontró con Carlos Büchler, un ex miembro de la CNEA que trabaja para $A r$ gonne Nacional Laboratory en los EE. UU. y una simple frase cambió el rumbo del desarrollo nuclear argentino.

"Hágame caso, Ingeniero, vaya a conocer el Argonauta" fue la frase que le dijo Carlos Büchler." (CNEA, 2008:35)

Quihillalt viajó a Chicago para ver el reactor de investigación, Argonaut. Era un modelo de baja potencia, solamente $10 \mathrm{Kw}$, de bajo costo y suficientemente seguro para el entrenamiento de los estudiantes de la Universidad de Chicago. Domingo (2003) asegura que Büchler convenció a Quihillalt de que el reactor podría ser replicado en la Argentina.

Sin mediar tiempo, Quihillat se entrevistó con John Hall en Washington D.C., encargado de las relaciones exteriores de la USAEC. No solo se obtuvo el permiso y los planos para replicar el reactor, sino también la posibilidad de enviar técnicos de la CNEA a capacitarse a Argonne Nacional Laboratory, trabajando directamente con el Argonaut.

En la CNEA los planos adquiridos fueron estudiados en detalle y se encontraron varios errores en el diseño original, como el blindaje de la tapa superior, y el listado de comprobación de arranque del reactor. Como consecuencia de estas advertencias desde la CNEA al Argonne Nacional Laboratory, el Director del reactor, Harry Bryant recibió cordialmente a los tres técnicos enviados, Fidel Alsina, Miguel Geiger y Carlos Domingo a quienes trató con deferencia. Los argentinos habían demostrado con sus conocimientos estar a la altura necesaria para ser considerados iguales. Como consecuencia, fueron invitados a las pruebas especiales del reactor, en donde se lo sometía a máxima potencia, pruebas con agua y cambios de disposición de las placas de uranio.

Durante el entrenamiento en Estados Unidos, el equipo de la CNEA seguía la construcción de la copia del reactor $A r$ gonaut. Se enviaron unos reportes sobre pruebas de combustible que se habían desarrollado en Argentina. La calidad de la misma era superior a la que sería provista por la empresa estadounidense. Bryant los alentó a que dieran de baja ese suministro y utilizaran el combustible propio que podían desarrollar en Argentina.

"El 31 de octubre la CNEA firmó el contrato en Washington con la US AEC para adquirir los seis kilogramos de uranio 238 enriquecido al $20 \%$ en uranio 235 , cantidad estipulada por el acuerdo de cooperación firmado en 1955, que aseguraba la provisión de combustible necesaria para el reactor de investigación argentino. [...]
De acuerdo con Quihillalt, era la primera vez que EE. UU. exportaba materia prima para que otro país elaborara sus propios elementos combustibles." (HURTADO, 2014:86)

Al finalizar el entrenamiento, los tres técnicos de la CNEA regresaron a Argentina en noviembre de 1957. La construcción del reactor se encontraba en su etapa final. Se prosiguió con las instalaciones y puesta a punto, trabajando entre 12 y 18 horas diarias, según relata Domingo (2003).

En Enero de 1958, comenzaron con las pruebas de puesta a crítico del reactor. La misma se hizo con extrema lentitud para evitar que el reactor tomara una potencia que no pudiera ser controlada. Se iba agregando uranio en dosis pequeñas y se esperaba que éste se estabilizara antes de continuar con el mismo procedimiento. En un punto del experimento se percataron de que el uranio disponible no sería suficiente para que el reactor tomara estado crítico. La solución fue alterar la geometría del uranio, cambiar de lugar las placas de uranio sin detener el reactor. Gracias a esa acción, el 17 de enero de 1958 a las 6:25hs. el reactor RA-1 alcanzó estado crítico.

El 20 de enero de 1958 se inauguró oficialmente el primer reactor construido y en funcionamiento de América Latina. Finalmente, Argentina había demostrado tener la capacidad tecnológica y científica para ingresar al predilecto club nuclear.

"En cuanto al significado tecnológico del RA-1, Quihillalt interpretó que "este modesto reactor" era un primer paso hacia los reactores de potencia "como uno de los modos que pueden concurrir a conjugar el déficit energético en el porvenir". También enfatizó que habian participado 32 empresas argentinas en su construcción.

El día posterior a la inauguración del RA-1, una noticia de apenas nueve líneas en The New York Times anunciaba: "El presidente Pedro Eugenio Aramburu encendió el primer reactor atómico en América Latina". (HURTADO, 2014:87)

Ornstein (2010) llama al período entre 1950 y 1958, la etapa formativa de la CNEA, cerrando la misma con la activación del RA-1. Lo cual demostraba también, que Argentina había optado por un camino diferente de desarrollo nuclear, no había comprado como la mayoría de los países un reactor llave en mano, sino que había logrado construir gran parte del mismo en el país. Esta situación también indicaría la dirección futura de la CNEA: un desarrollo autónomo, lejos 


\section{El cambio en la política exterior de Estados Unidos que permitió el desarrollo del uso pacífico de energía nuclear en Argentina}

de la dependencia tecnológica de los países centrales, pero integrado a la comunidad internacional.

"El sentido simbólico del RA-1 se completaba con la venta del know-how desarrollado en el proceso de fabricación de sus elementos combustibles a la empresa alemana Degussa. Este desarrollo había sido presentado, en agosto de 1958, en la Segunda Conferencia Internacional sobre Usos Pacíficos de la Energía Atómica. La venta se concretó en noviembre en Frankfurt, donde la CNEA entregó un informe completo del proceso de fabricación a cambio de la suma de 14.000 dólares. Esta transferencia era la primera exportación de tecnología nuclear de la Argentina." (HURTADO, 2014:91)

\section{Consideraciones finales}

Argentina como país de América Latina puede ser clasificado como un país subdesarrollado en términos de su economía interna; periférico en relación a que su economía agroexportadora y; dependiente debido a que muchas veces sus políticas internas han sido influenciadas desde el exterior a través de lineamientos que condicionan sus decisiones a cambio del acceso a créditos internacionales.

Pero, en el área nuclear, Argentina escogió un camino completamente alternativo. A diferencia de la mayorías de los países periféricos que compraron tecnología llave en mano, colocándose en una nueva posición de dependencia tecnológica, Argentina transitó por un camino largo -no si complicaciones como hemos visto-, pero que le otorgó la autonomía para determinar su propio rumbo.

Argentina, al lograr desarrollar su propio reactor nuclear fue reposicionada dentro del espectro del sistema mundo. En temas nucleares es un país semiperiférico con gran influencia y relaciones con los países centrales. Incluso, fue capaz de invertir roles al conseguir que los Estados Unidos exportaran uranio para que el país produjera su propio combustible. Es la inversión de la exportación de materia prima de un país central a uno periférico. Además, Alemania adquirió un producto de valor agregado al comprar los reportes sobre la producción de combustible, alterando nuevamente el orden tradicional de país periférico.

Pero, el desarrollo de la energía nuclear no puede estar desvinculado del cambio en la cooperación internacional tanto de la política exterior de Estados Unidos, el cambio del secretismo de la Administración Truman a una cooperación abierta con la Administración Eisenhower, indistintamente de la veta comercial. Y a las propias relaciones entre Argentina y los Estados Unidos, de una tensión y aislamiento durante la primera presidencia de Perón, la cual -por motivos económicos- comenzó a acercarse nuevamente hacia el país del norte y se fue acrecentando con los diferentes gobiernos, De Factos y Civiles. Toda esta situación derivó en que se autorizara el uso de los planos del Argonaut, la cancelación sin represalias de la compra del reactor y del combustible a empresas estadounidenses, y la venta de los primeros componentes de uranio. Si una relación pacífica entre ambas naciones, no hubiera sido posible la existencia del reactor propio. 


\section{El cambio en la política exterior de Estados Unidos que permitió el desarrollo del uso pacífico de energía nuclear en Argentina}

\section{Bibliograffia}

BALSEIRO, J.A. (1952). Informe del Dr. José Antonio Balseiro referente a la inspección realizada en la isla Huemul en setiembre de 1952. En: II Seminario Regional de Física Forense 2001. Bariloche. Disponible en: http://fisica.cab.cnea.gov.ar/forense/publicaciones/semin 2001/ balseiro.pdf Consulta: 28 de Julio de 2016.

CNEA (2008). A 50 años de la inauguración del RA-1, un hito en el desarrollo nuclear argentino. CNEA: Buenos Aires. Año 8, Número 29/30, Enero-Junio 2008, pp. 35-36. Disponible en: http://www.cnea.gov.ar/ sites/default/files/ra1 $0 . p d f$ Consulta: $1^{\circ}$ de agosto de 2016.

Decreto 10.936. Presidencia de la Nación. Buenos Aires, 31 de mayo de 1950. Disponible en: http://servicios.infoleg.gob.ar/infolegInternet/ anexos/195000-199999/198653/norma.htm Consulta: 29 de Julio de 2016.

DOMINGO, C. (2003). Breve historia del reactor RA1. CNEA: Buenos Aires. Año 3, Número 11/12, Julio-Diciembre 2003, pp. 29-31. Disponible en: http://www.cnea.gov.ar/sites/default/files/RA1.pdf Consulta: $1^{\circ}$ de agosto de 2016.

DOMINGUES, J. M. (2012). Desarrollo, periferia y semiperiferia en la tercera fase de la modernidad global. CLACSO, Ciudad Autónoma de Buenos Aires.

EISENHOWER, D. (1953). Text of the address delivered by the President of the United States before the General Assembly of the United Nations in New York City. The Dwight D. Eisenhower Library, Washington D.C., EE.UU. Disponible en: https://www.eisenhower.archives. gov/research/online documents/atoms for peace/Binder13.pdf Consulta: 2 de agosto de 2016.

FREIDEL F. y SIDEY, H. (2006). The Presidents of the United States of America. White House Historical Association, Washington D. C., EE. UU.

HASEGAWA, T. (2007).The Atomic Bombs and the Soviet Invasion: What Drove Japan's Decision to Surrender?. Volume 5, Issue 8, Number 0. The Asia-Pacific Journal, Stanford University, California, EE.UU.

HURTADO, D. (2014). El sueño de la Argentina atómica: Política, tecnología nuclear y desarrollo nacional (1945-2006). Edhasa: Buenos Aires.

LEVINE, P. (?). A Photo-Essay on the Bombing of Hiroshima and Nagasaki. University of Illinois. Disponible en: http://www.english.illinois.edu/ maps/poets/g_l/levine/bombing.htm Consulta: 2 de agosto de 2016.
MIZELLE, W. (1947a). Exclusive - Peron's Atomic Plans. En: The New Republic. 24 de febrero de 1947.

- (1947b). More About Peron's Atom Plans. En: The New Republic. 31 de marzo de 1947.

MORLAND, H. (2005). Born Secret. En: Cardozo Law Review, Vol. 26, Nro. 4. Washington D. C., EE.UU. March. pp. 1401-1408. Disponible en: https://fas.org/sgp/eprint/cardozo.pdf Consulta: 28 de julio de 2016.

ORNSTEIN, R. (2010). El desarrollo nuclear argentino: 60 años de una historia exitosa. CNEA: Buenos Aires. Año 10, Número 37/38, Enero-Junio 2010, pp. 6-14. Disponible en: http://www.cnea.gov.ar/sites/ default/files/60anios.pdf Consulta: 3 de agosto de 2016.

Public Law 585: Atomic Energy Law. US Congress. Washington D. C., EE. UU., 1946. Disponible en: https://science.energy.gov/ /media/bes/ pdf/Atomic Energy Act of 1946.pdf Consulta: 28 de julio de 2016.

SHALETT, S. (1945).First Atomic Bomb Dropped on Japan; Missile Is Equal to 20,000 Tons of TNT; Truman Warns Foe of a 'Rain of Ruin'. En: The New York Times. Vol. XCIV, Nro. 31.972. 7 Agosto 1945. Disponible en: http://www.nytimes.com/learning/general/onthisday/big/0806. html\#article Consulta: 2 de agosto de 2016.

WALLERSTEIN (1984).El moderno sistema mundial II. El mercantilismo y la consolidación de la economía-mundo europea, 1600-1750. Siglo XXI Editores: México.

- (2005).The Modern World System in Crisis: Bifurcation, Chaos and Choices.En: World System Analysis. An Introduction, Duke University Press, 2nd. Edition, 2005, pp.76-90 